\title{
INTERCONNECTION OF PERFECTION AND SUCCESS IN LEARNING FOREIGN LANGUAGES
}

\author{
Olga Yu. Savina
}

University of Tyumen,

6 Volodarskogo Street, 625003 Tyumen, Russia.

\begin{abstract}
The article deals with the relationship between perfectionism in the academic environment and foreign language learning. After defining the basic concepts (the three-dimensional model of perfectionism, its components, the multidimensional perfectionism scale by P. Hewitt and G. Flett), the results of the current related research conducted abroad and in Russia are briefly presented. The literature review allowed us to formulate a research question: Are the foreign language and its level of proficiency (on the Common reference levels) related to the degree of perfectionism and its components?

In search of an answer to this question, an empirical study was conducted (sample size 213 participants). Using objective statistical methods, it was found that, in general, there are no statistically significant differences between students studying different languages and students at different levels. However, differences in the degree of perfectionism between A2 level and neighboring levels attract attention in terms of the effect sizes. For individual components of perfectionism, the levels of Basic user (A1 and A2) differ most from other levels.

The presented results can be useful both for foreign language teachers and for scientists dealing with the success of language learning and the factors affecting this process.
\end{abstract}

Key Words: self-oriented perfectionism, other-oriented perfectionism, socially prescribed perfectionism, foreign language proficiency, self-assessment

For citation: Savina O.Yu. 2020. Interconnection of Perfection and Success in Learning Foreign Languages. Philological Sciences at MGIMO. Vol. 6. No 4(24). P. 112-124. https://doi.org/10.24833/24102423-2020-4-24-112-124

\section{ВЗАИМОСВЯЗЬ ПЕРФЕКЦИОНИЗМА И УСПЕШНОСТИ ИЗУЧЕНИЯ ИНОСТРАННЫХ ЯЗЫКОВ}

О.Ю. Савина

Тюменский государственный университет, 625003, Россия, Тюмень, ул. Володарского, 6 
Аннотация. Статья посвящена вопросу о взаимосвязи между перфекиионизмом в академической среде и изучением иностранного языка. После определения основных понятий (трёхмерная модель перфекиионизма, её составляющие, многомерная шкала перфекиионизма П. Хьюитта и Г. Флетта) кратко представлены результаты актуальных сменных исследований, проведённых за рубежом и в России. Литературный обзор позволил выявить лакунь, требующие внимания, и сбормулировать исследовательский вопрос: Связаны ли изучаемый иностранный язык и уровень владения им (по общеевропейской шкале) со степенью выраженности перфекиионизма и его составляющих?

В поисках ответа на этот вопрос было проведено эмпирическое исследование (объём выборки 213 человек). С использованием объективных статистических методов было выявлено, что статистически значимых различий между студентами, изучающими разные языки и владеющими ими на разных уровнях, в иелом нет. Однако по силе эффекта обрашают на себя внимание отличия в степени перфекиионизма между уровнем А2 и соседними уровнями. По отдельным составляющим перфекиионизма наиболее сильно отличаются уровни элементарного владения (А1 и А2) от других уровней.

Представленные результаты могут быть полезны как преподавателям иностранных языков, так и учёным, занимающимся вопросами успешности изучения языков и факторов, оказывающих влияние на этот процесс.

Ключевые слова: перфекиионизм, ориентированный на себя; перфекиионизм, ориентированныцй на других; социально предписанный перфекционизм, иностранный язык, уровень владения, самооценка

Для цитирования: Савина О.Ю. 2020. Взаимосвязь перфекционизма и успешности изучения иностранных языков. Филологические науки в МГИМО. Том 6. № 4(24). С. 112-124. https://doi. org/10.24833/2410-2423-2020-4-24-112-124

\section{Введение}

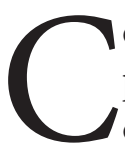
овременные эмпирические исследования в области лингводидактики традиционно опираются на разные базовые науки, включая психологию, дидактику и лингвистику [12, c. 127-128]. Однако проблема перфекционизма в академической среде в целом и его влияния на успеваемость по иностранным языкам в частности изучена по-прежнему недостаточно глубоко [5; 20; 10].

Как перфекционизм, то есть стремление быть совершенным, безупречным во всем, определяет успешность изучения иностранных языков? На первый взгляд может показаться, что перфекционисты, стремясь к совершенству, всё делают лучше. Принято считать, что перфекционизм присущ академически успешным и одарённым людям, прилежным ученикам. Значит ли это, что и иностранный язык перфекционисты могут освоить лучше других? Мы предположили, что не только перфекционизм как свойство человеческой личности влияет на эффективность изучения иностранного языка, но и сам изучаемый язык накладывает своеобразный отпечаток на личность изучающего, то есть перфекционизм и иностранный язык оказывают друг на друга взаимное влияние.

В статье представлены различные точки зрения на перфекционизм и его виды, проанализированы исследования перфекционизма в академической среде, проведённые в последние годы, а также подробно изложены результаты собственного эмпирического исследования, нацеленного на определение характера взаимосвязи между перфекционизмом и изучаемым иностранным языком, и успешностью его изучения, представленной самооценкой по общеевропейской шкале.

\section{1. Виды перфекционизма}

Существует много разных подходов к пониманию понятия перфекционизма и его структуры. В самом широком толковании под перфекционизмом понимается «стремление к безупречности и совершенству в поведении и деятельности, касающихся разных сфер жизни» $[8$, с. 66]. Несмотря 
на существующие разные точки зрения, большинство учёных сходятся во мнении, что перфекционизм включает в себя, во-первых, «перфекционистское стремление» (perfectionistic strivings) высокие личные стандарты (ожидаемые результаты), а во-вторых, «перфекционистские проблемы» (perfectionistic concerns) - чрезмерную самокритику или опасения по поводу достижения или поддержания личных стандартов. Приведённые термины принадлежат Й. Штёберу и К. Отто [17, с. 368]. Перфекционистское стремление как требовательное совершенство себя и установление высоких личных стандартов некоторыми исследователями оценивается как позитивное (или адаптивное), в то время как перфекционистские проблемы рассматриваются как тенденция к чрезмерной фокусировке на недостатках, ошибках, критике и воспринимаемом несоответствии между фактическими и идеальными достижениями [20, с. 174].

Разными психологическими школами были разработаны несколько концепций перфекционизма: американская (R. Slaney, J. Ashby, K. Rice), канадская (P. Hewitt, G. Flett), британская (R. Frost) и российская (Н.Г. Гаранян, А.Б. Холмогорова, Т.Ю. Юдеева [5, с. 429]. Подробный анализ существующих моделей перфекционизма хорошо представлен В.А. Ясной и С.Н. Ениколоповым [9].

В интересах данного исследования остановимся на двух подходах: противопоставлении адаптивного / неадаптивного перфекционизма и выделении перфекционизма, ориентированного на себя, на других, и социально предписанного перфекционизма.

Деление на положительный (позитивный, здоровый, адаптивный) и отрицательный (негативный, патологический, неадаптивный) перфекционизм поддерживается многими исследователями $[16 ; 15 ; 7]$. «Нездоровые» перфекционисты имеют более высокий уровень перфекционистских стремлений, но низкий уровень забот, а «здоровые» перфекционисты имеют высокий уровень стремлений, но низкий уровень забот [17, с. 368]. Иными словами, отрицательный перфекционизм нацелен на предотвращение негативных последствий своих действий / бездействия, а положительный - на достижение позитивных последствий [16, с. 667].

В настоящее время активно применяется и трёхмерная модель перфекционизма, разработанная П. Хьюиттом и Г. Флеттом [18], в рамках которой выделяют перфекционизм, ориентированный на себя (ПОС, self-oriented perfectionism), перфекционизм, ориентированный на других (ПОД, other-oriented perfectionism), и социально предписанный перфекционизм (СПП, socially prescribed perfectionism).

Перфекционизм, ориентированный на себя, подразумевает нереалистичные, завышенные требования, предъявляемые к себе, самокопание и самокритику, которые делают невозможным принятие собственных изъянов, недостатков и неудач. Перфекционизм, ориентированный на других, подразумевает преувеличенные и нереалистичные требования к окружающим. Социально предписанный перфекционизм - это ощущение человека, что ему предъявляют завышенные и нереалистичные требования, которым необходимо соответствовать, хотя это и нереально [2].

Эта трёхмерная модель перфекционизма будет применена в данном исследовании в связи с тем, что авторов интересуют разнонаправленные факторы, определяющие успех в изучении иностранных языков. Кроме того, трёхмерная модель позволяет учесть не только субъектно-ориентированный, но и объектно-ориентированный перфекционизм.

Для диагностики перфекционизма разработано большое количество инструментов (подробнее см. у А.А. Золотаревой [3]). В настоящем исследовании была использована «Многомерная шкала перфекционизма» (Multidimensional Perfectionism Scale, MPS) П. Хьюитта (P.L. Hewitt) и Г. Флетта (G.L. Flett) [6], впервые адаптированная в России И.И. Грачевой [1]. Она содержит несколько подшкал и позволяет выявить степень и характер соотношения составляющих перфекционизма.

\section{2. Исследования перфекционизма в академической среде}

В последние годы перфекционизм в академической среде всё чаще становится предметом исследований в разных странах мира и в разных академических контекстах. Так, было установлено, что любое увеличение положительных показателей перфекционизма у студентов связано со снижением уровня депрессии, а также с повышением их самоценки (Иран, 2010) [19, с. 34]. Была 
выявлена положительная взаимосвязь между многомерным перфекционизмом и мотивационной ориентацией, как внутренней, так и внешней (Иордания, 2019) [13, с. 107-108]. При сравнении двух разных возрастных групп и академических контекстов - студентов университета и учеников гимназии - было установлено, что фактические достижения учащихся не зависят от перфекционистского профиля и самооценки (Финляндия, 2019) [14].

Исследований о закономерностях проявления перфекционизма при изучении иностранных языков крайне мало, и многие учёные отмечают, что этот вопрос требует ответа [15, с. 136; 10 , c. 95]. Приведём три примера.

Л.Б. Киселёва (СПбГМТУ, 2015) определяла зависимость успеваемости по английскому языку от выраженности у студентов перфекционизма (на первом курсе технического вуза) и установила, что самые высокие показатели перфекционизма оказались у относительно успешных в английском языке студентов (в отличие от успешных и неуспешных) [5, с. 432], то есть «студенты с высоким уровнем перфекционизма редко достигают значительных успехов в овладении иностранным языком» $[5$, с. 437].

К. Ванг, Т.М. Пермякова и М.С. Шевелева (ВШЭ, 2018) исследовали взаимосвязь между перфекционизмом и языковой тревожностью при изучении иностранного языка. Авторы пришли к выводу, что такая связь существует. Возможное объяснение этой взаимосвязи они видят в том, что перфекционисты, как правило, озабочены своими ошибками в иностранном языке, которые для них означают несовершенство. Для них ограниченное знание о лексике и грамматике нового языка может быть очень проблематичным. Ведь изучение языка означает многократное повторение ошибок и недостатков. Таким образом, авторы рассматривают перфекционизм как потенциальный барьер для изучения иностранных языков [15].

Исследуя взаимодействие между эффективностью изучения английского языка и перфекционизмом, И. Фараг (США, 2020) установил, что уровень владения изучаемым языком не коррелирует с самооценкой [10].

Таким образом, проведённый анализ литературы позволяет сделать вывод, что в России было мало исследований перфекционизма при изучении иностранных языков. Сравнительных исследований при изучении разных языков вообще не удалось обнаружить. В то время как необходимость сравнения того, как влияет перфекционизм на процесс изучения разных иностранных языков, отмечается другими учеными [15, с. 145-146]. Помимо этого, авторы подчёркивают, что нужно больше узнать о различных факторах и психологических механизмах, связанных с успешным освоением иностранных языков.

\section{3. Эмпирическое исследование}

Таким образом, концепция данного исследования вполне соответствует актуальной научной повестке. Его гипотеза состояла в том, что перфекционизм может иметь разную степень выраженности у студентов, изучающих разные иностранные языки как специальность (так как каждая языковая система накладывает свой ментальный отпечаток), а также у студентов, владеющих этими языками на разном уровне.

Для проверки этой гипотезы было проведено эмпирическое исследование. Участниками выборки стали 213 студентов первого-четвертого курса бакалавриата Института социально-гуманитарных наук Тюменского государственного университета (32 юноши, 181 девушка). Большинство студентов учатся на направлении Лингвистика (173 человека), то есть изучают языки как специальность - интенсивно и глубоко, остальные - на других направлениях (40 человек), то есть изучают языки только как общеобразовательную дисциплину. Студенты принимали участие в исследовании добровольно и анонимно. Им была объяснена цель исследования, которое включало анкетирование о демографических данных и опросник, построенный на Многомерной шкале перфекционизма П. Хьюитта и Г. Флетта. Опрос проводился в электронном формате с использованием инструмента Google.Forms.

Для сбора эмпирических данных была использована Многомерная шкала перфекционизма (Multidimensional perfectionism scale; МШП). Данный опросник состоит из 45 вопросов (по 
15 баллов в каждой из трёх шкал), время прохождения теста 10-15 минут. Сумма баллов, набранная испытуемым по всем трём субшкалам, отражает общую степень перфекционизма [2].

Для обработки данных использовался табличный редактор Excel v. 2010, статистический пакет SPSS Statistics v. 23 и программа JASP v. 0.11.1.

\section{4. Результаты исследования}

Собранные данные были проанализированы - проведено сравнение выявленных уровней перфекционизма и его составляющих по изучаемым языкам и по самооценке уровня владения иностранным языком.

В целом по выборке преобладает средний уровень общего перфекционизма, то есть умеренно выраженное стремление быть совершенным и безупречным во всём (67\%) (см. рис. 1). Этот результат согласуется с данными, приведёнными Н.Н. Карловской и А.С. Сысоевой $[4$, с. 6].

Если рассматривать отдельно различные виды перфекционизма, то можно заметить, что перфекционизм, ориентированный на себя (ПОС), выражен наименее сильно. В то время как перфекционизм, ориентированный на других (ПОД), и социально предписанный перфекционизм (СПП) выражены гораздо сильнее. По-видимому, даже при среднем уровне общего перфекционизма современные студенты более требовательны к другим, чем к себе, и более чувствительны к требованиям общества.

\section{Уровни перфекционизма и его вндов}

(в процентах ко всей выборке)

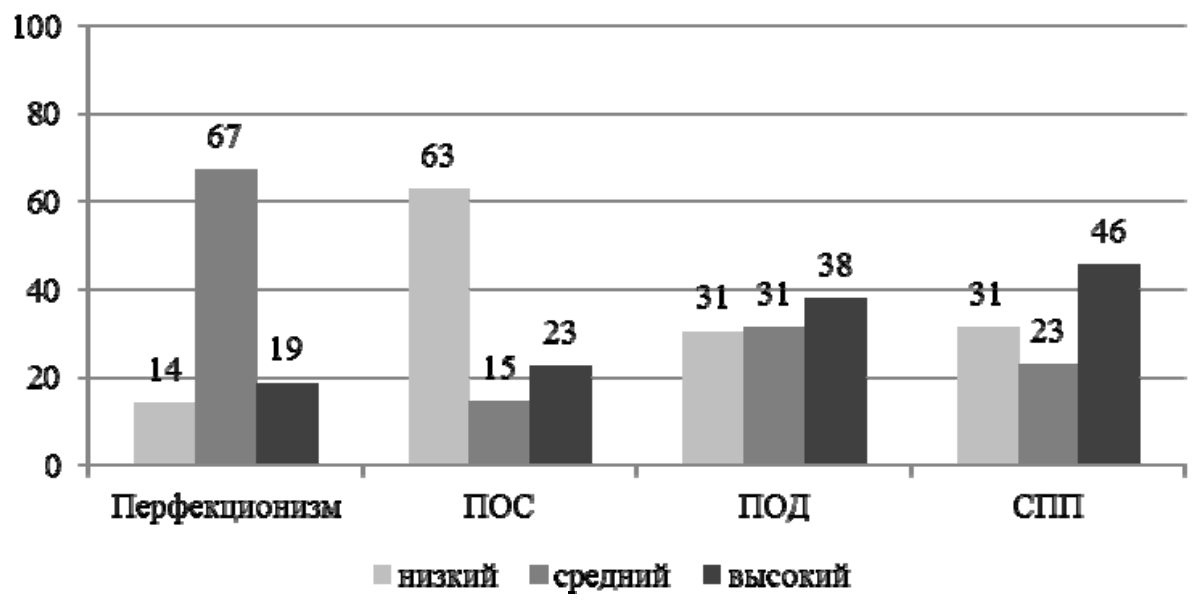

Рис. 1. Уровни перфекционизма и его видов (в целом по выборке)

Таблица 1.

Описательные статистики групп по изучаемым языкам

\begin{tabular}{|c|c|c|c|c|c|c|c|c|c|}
\hline $\begin{array}{l}\text { Виды } \\
\text { перфекционизма }\end{array}$ & ия & $\mathrm{N}$ & Среднее & Медиана & Ст. отклонение & Шапиро-Уилка & $\begin{array}{c}\text { Уровень значимости } \\
\text { Шапиро-Уилка }\end{array}$ & Минимум & Максимум \\
\hline \multirow{3}{*}{$\begin{array}{l}\text { Перфекционизм } \\
\text { общий }\end{array}$} & en & 135 & 184,59 & 186,2 & 24,82 & 0,99 & 0,17 & 95,2 & 259 \\
\hline & de & 46 & 188,73 & 184,8 & 26,19 & 0,96 & 0,07 & 134,4 & 257,6 \\
\hline & $\mathrm{fr}$ & 32 & 181,13 & 182 & 16,2 & 0,93 & 0,04 & 154 & 229,6 \\
\hline \multirow{3}{*}{$\begin{array}{l}\text { Перфекционизм, } \\
\text { ориентированный } \\
\text { на себя }\end{array}$} & en & 135 & 68,98 & 67,2 & 13 & 0,99 & 0,55 & 30,8 & 103,6 \\
\hline & de & 46 & 69,27 & 69,3 & 13,93 & 0,99 & 0,83 & 37,8 & 100,8 \\
\hline & $\mathrm{fr}$ & 32 & 68,60 & 68,6 & 10 & 0,98 & 0,7 & 51,8 & 91 \\
\hline \multirow{3}{*}{$\begin{array}{l}\text { Перфекционизм, } \\
\text { ориентированный } \\
\text { на других }\end{array}$} & en & 135 & 57,03 & 57,4 & 10,74 & 0,98 & 0,04 & 21 & 91 \\
\hline & de & 46 & 57,22 & 56 & 10,5 & 0,97 & 0,3 & 37,8 & 81,2 \\
\hline & $\mathrm{fr}$ & 32 & 55,26 & 56 & 7,01 & 0,96 & 0,34 & 42 & 70 \\
\hline \multirow{3}{*}{$\begin{array}{l}\text { Социально } \\
\text { предписанный } \\
\text { перфекционизм }\end{array}$} & en & 135 & 58,58 & 58,8 & 10,29 & 0,99 & 0,2 & 30,8 & 95,2 \\
\hline & de & 46 & 62,24 & 61,6 & 10,14 & 0,97 & 0,19 & 44,8 & 88,2 \\
\hline & $\mathrm{fr}$ & 32 & 57,27 & 56 & 8,11 & 0,93 & 0,03 & 44,8 & 84 \\
\hline
\end{tabular}




\section{1. Сравнение уровней перфекционизма по изучаемым языкам}

Данные по изучаемым языкам представлены в таблице 1. Независимая переменная Изучаемый язык является номинальной шкалой и включает три уровня: английский - немецкий - французский. Группы полагаются как несвязанные, потому что каждый студент опрашивался только в отношении одного изучаемого языка.

Средний уровень общего перфекционизма характеризует и каждую из языковых групп (en $-65 \%$, de $-65 \%$, fr - 78\%). Это же можно сказать и о перфекционизме, направленном на себя (en $-64 \%$, de $-54 \%$, fr - 72\%). В то время как перфекционизм, направленный на других, заметно отличается (см. рис. 2).

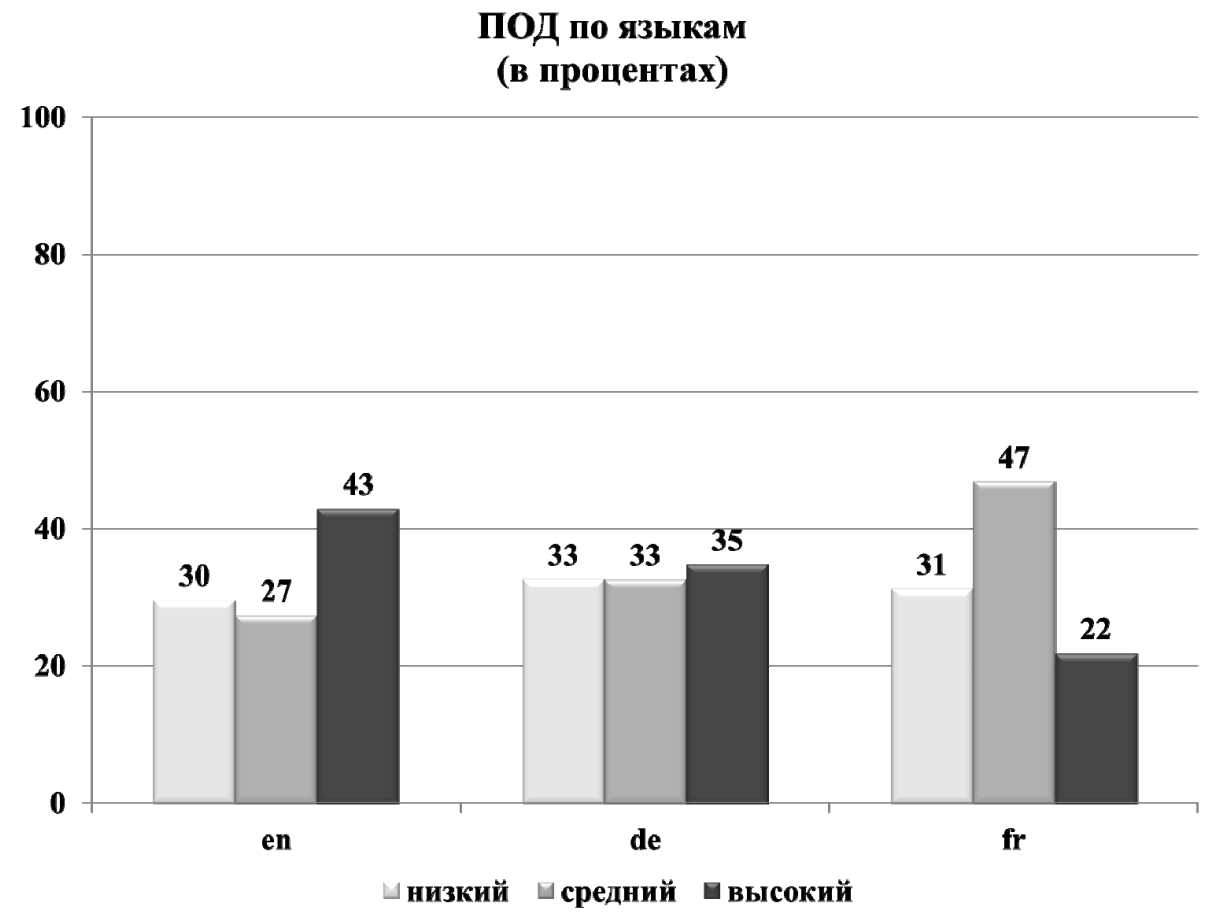

Рис. 2. Перфекционизм, ориентированный на других, по языкам (в процентах)

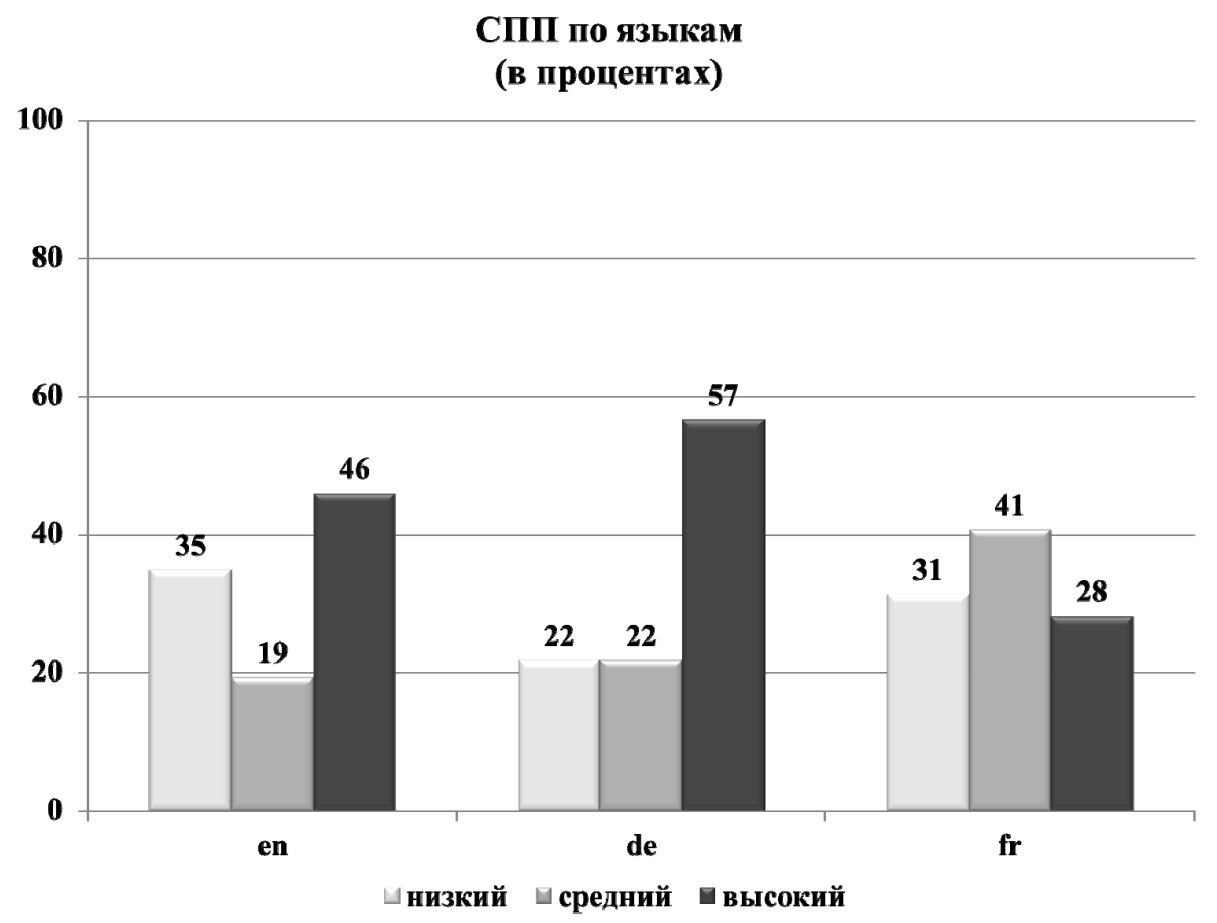

Рис. 3. Социально предписанный перфекционизм по языкам (в процентах) 
Мы видим, что «англичане» более требовательны к окружающим. Среди них доля высокого уровня перфекционизма, ориентированного на других, в два раза выше, чем среди «французов». «Немцы» же выделяются тем, что равномерно распределились в своих требованиям к окружающим.

В то же время «немцы» наиболее остро ощущают социально предписанный перфекционизм (см. рис. 3).

Таким образом, визуальное сравнение групп по изучаемым языкам показывает некоторые отличия, значимость которых подлежит проверке с использованием статистических методов.

\section{2. Сравнение уровня перфекционизма по самооценке уровня владения иностранным языком}

Данные по самооценке уровня владения изучаемым языком представлены в таблице 2. Независимая переменная является порядковой шкалой и включает пять уровней (A1 - A2 - B1 - B2 и C1-C2). Уровни C1 и С2 пришлось объединить (так как на уровне С2 мало наблюдений), от категории «Затрудняюсь ответить» пришлось отказаться (также из-за малого количества наблюдений). Группы несвязанные.

Таблица 2.

Описательные статистики групп

по самооценке уровня владения изучаемым иностранным языком

\begin{tabular}{|c|c|c|c|c|c|c|c|c|c|}
\hline $\begin{array}{l}\text { Виды } \\
\text { перфекционизма }\end{array}$ & $\begin{array}{l}\text { Самооценка } \\
\text { уровня владения } \\
\text { изучаемым языком }\end{array}$ & $N$ & Среднее & Медиана & $\begin{array}{l}\text { Ст. } \\
\text { отклонение }\end{array}$ & $\begin{array}{l}\text { Шапиро- } \\
\text { Уилка }\end{array}$ & $\begin{array}{l}\text { Уровень } \\
\text { значимости } \\
\text { Шапиро-Уилка }\end{array}$ & Минимум & Максимум \\
\hline \multirow{5}{*}{$\begin{array}{l}\text { Перфекционизм } \\
\text { общий }\end{array}$} & A1 & 8 & 186,55 & 184,8 & 14,43 & 0,87 & 0,17 & 170,8 & 217 \\
\hline & $\mathrm{A} 2$ & 21 & 178,6 & 177,8 & 16,93 & 0,96 & 0,56 & 154 & 215,6 \\
\hline & B1 & 40 & 187,92 & 188,3 & 20,91 & 0,92 & 0,01 & 158,2 & 257,6 \\
\hline & B2 & 95 & 184,21 & 184,8 & 27,16 & 0,99 & 0,86 & 123,2 & 259 \\
\hline & $\mathrm{C} 1-\mathrm{C} 2$ & 43 & 186,36 & 182 & 25,42 & 0,92 & 0,01 & 95,2 & 229,6 \\
\hline \multirow{5}{*}{$\begin{array}{l}\text { Перфекционизм, } \\
\text { ориентированный } \\
\text { на себя }\end{array}$} & A1 & 8 & 69,83 & 72,1 & 9,21 & 0,95 & 0,73 & 53,2 & 81,2 \\
\hline & A2 & 21 & 65,4 & 64,4 & 7,62 & 0,91 & 0,07 & 54,6 & 77 \\
\hline & B1 & 40 & 72,59 & 68,6 & 12,31 & 0,95 & 0,05 & 51,8 & 99,4 \\
\hline & B2 & 95 & 67,94 & 68,6 & 13,43 & 0,99 & 0,91 & 36,4 & 103,6 \\
\hline & $\mathrm{C} 1-\mathrm{C} 2$ & 43 & 70,13 & 70 & 13,98 & 0,98 & 0,52 & 30,8 & 98 \\
\hline \multirow{5}{*}{$\begin{array}{l}\text { Перфекционизм, } \\
\text { ориентированный } \\
\text { на других }\end{array}$} & A1 & 8 & 52,5 & 53,2 & 9,38 & 0,97 & 0,93 & 37,8 & 67,2 \\
\hline & A2 & 21 & 54,73 & 56 & 8,93 & 0,9 & 0,04 & 43,4 & 81,2 \\
\hline & B1 & 40 & 55,51 & 54,6 & 9,28 & 0,98 & 0,71 & 30,8 & 77 \\
\hline & B2 & 95 & 57,49 & 57,4 & 10,93 & 0,98 & 0,13 & 26,6 & 91 \\
\hline & $\mathrm{C} 1-\mathrm{C} 2$ & 43 & 58,08 & 60,2 & 10,15 & 0,89 & 0,001 & 21 & 72,8 \\
\hline \multirow{5}{*}{$\begin{array}{l}\text { Социально } \\
\text { предписанный } \\
\text { перфекционизм }\end{array}$} & A1 & 8 & 64,23 & 62,3 & 10,3 & 0,94 & 0,62 & 50,4 & 84 \\
\hline & $\mathrm{A} 2$ & 21 & 58,47 & 58,8 & 6,87 & 0,97 & 0,77 & 47,6 & 71,4 \\
\hline & B1 & 40 & 59,82 & 58,8 & 9,31 & 0,94 & 0,03 & 44,8 & 88,2 \\
\hline & B2 & 95 & 58,79 & 57,4 & 11,28 & 0,97 & 0,02 & 30,8 & 95,2 \\
\hline & $\mathrm{C} 1-\mathrm{C} 2$ & 43 & 58,15 & 60,2 & 9,35 & 0,97 & 0,27 & 32,2 & 74,2 \\
\hline
\end{tabular}

Так как шкала уровней порядковая, то для этого сравнения помимо долей был использован линейный тренд, чтобы проследить, есть ли связь между уровнем владения языком и уровнем перфекционизма (см. рис. 4).

Мы видим, что чем выше уровень владения языком, тем выше степень общего перфекционизма. При этом тренд высокого уровня перфекционизма (тёмная линия) более явно выражен. Примерно так же обстоит дело с перфекционизмом, ориентированным на себя, и социально предписанным перфекционизмом. Однако в отношении требований к окружающим есть явное отличие (см. рис. 5) - высокая степень данного вида перфекционизма растёт заметно быстрее с ростом уровня языка (угол подъёма тёмной линии, в то время как две другие линии просто горизонтальны и практически совпадают). То есть студенты, требовательные к другим, становятся всё более требовательными, когда они растут в языке. 


\section{Перфекционизм повышается \\ вместе суровнем в.тадения языком \\ (в процентах)}

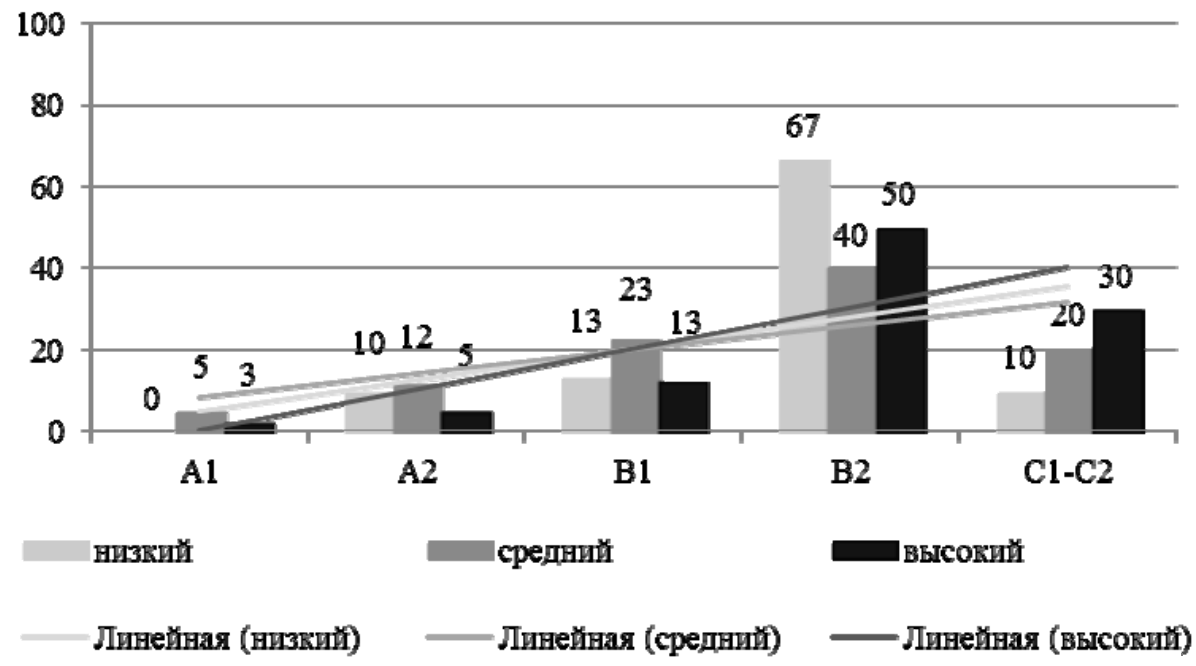

Рис. 4. Общий перфекционизм по уровню владения языком (самооценка)

Высокй уровень перфекщиониза, орнентированного на друтих, быстрее растет с повышением уровня в.тадения языком (в процентах)

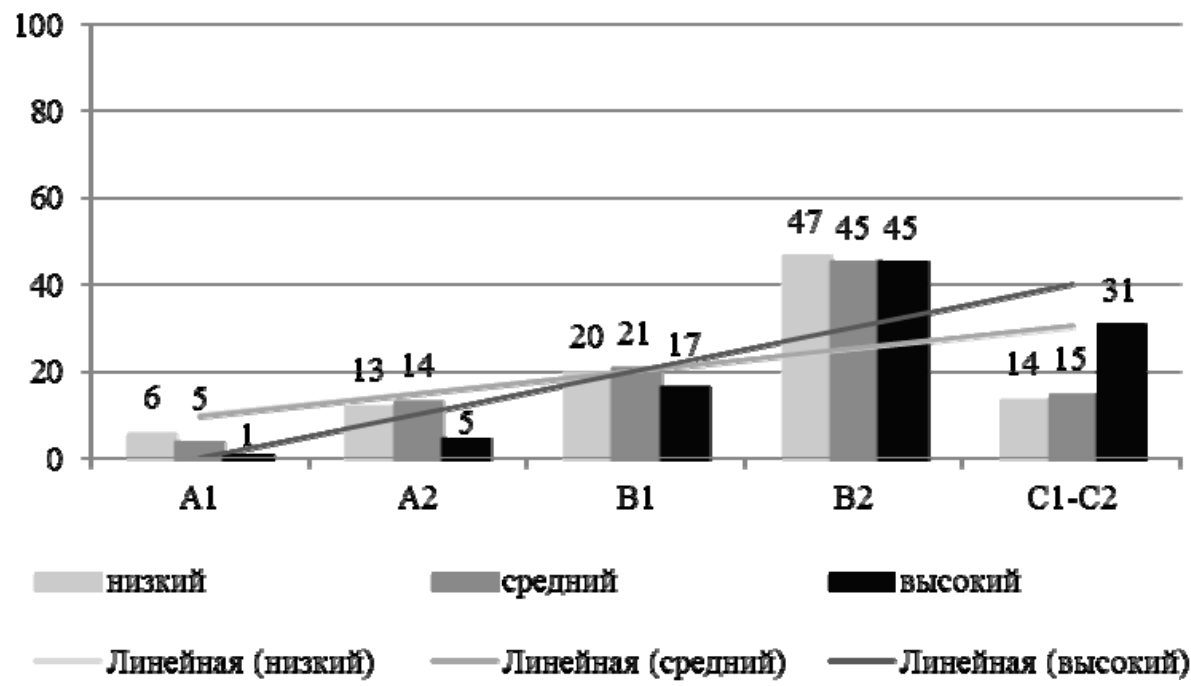

Рис. 5. Перфекционизм, ориентированный на других, по уровню владения языком (самооценка)

Значимость различий степени перфекционизма по уровням владения языком, выявленных при визуальном сравнении, также должна быть проверена статистическими методами.

\section{3. Статистическая проверка выявленных различий}

Соответствие полученных данных нормальному распределению было проверено графически и расчётным способом с использованием критерия Шапиро-Уилка. В большинстве групп по изучаемым языкам и по самооценке уровня владения языком распределение отличается от нормального.

Для проверки указанных различий был использован непараметрический критерий КраскелаУоллиса, так как группы несвязанные, а распределение отличается от нормального. Результаты вычисления эмпирического значения критерия представлены в таблице 3. 
Таблица 3.

Эмпирическое значение Н-критерия и р-уровня значимости

\begin{tabular}{|c|c|c|c|c|c|c|c|c|c|}
\hline \multirow{2}{*}{\multicolumn{2}{|c|}{\begin{tabular}{r|} 
Группа \\
$\mathrm{N}$ \\
\end{tabular}}} & en & de & $\mathrm{fr}$ & A1 & A2 & B1 & B2 & $\mathrm{C} 1-\mathrm{C} 2$ \\
\hline & & 135 & 46 & 32 & 8 & 21 & 40 & 95 & 43 \\
\hline \multirow[t]{3}{*}{ Перфекционизм общий } & Средний ранг & 107,96 & 112,1 & 95,63 & 107,69 & 84,81 & 109,96 & 102,56 & 110,33 \\
\hline & $\mathrm{H}$ & \multicolumn{3}{|c|}{1,438} & \multicolumn{5}{|c|}{3,119} \\
\hline & $p$ & \multicolumn{3}{|c|}{0,487} & \multicolumn{5}{|c|}{0,538} \\
\hline \multirow[t]{3}{*}{ ПОС } & Средний ранг & 107,40 & 107,40 & 107,40 & 111,81 & 84,02 & 117,60 & 100,12 & 108,23 \\
\hline & $\mathrm{H}$ & \multicolumn{3}{|c|}{0,033} & \multicolumn{5}{|c|}{5,155} \\
\hline & $p$ & \multicolumn{3}{|c|}{0,984} & \multicolumn{5}{|c|}{0,272} \\
\hline \multirow[t]{3}{*}{ под } & Средний ранг & 110,04 & 110,04 & 110,04 & 78,81 & 86,88 & 95,44 & 105,99 & 120,60 \\
\hline & $\mathrm{H}$ & \multicolumn{3}{|c|}{1,446} & \multicolumn{5}{|c|}{7,377} \\
\hline & $p$ & \multicolumn{3}{|c|}{0,485} & \multicolumn{5}{|c|}{0,117} \\
\hline \multirow[t]{3}{*}{ СПП } & Средний ранг & 104,23 & 104,23 & 104,23 & 133,63 & 103,02 & 107,75 & 100,34 & 103,57 \\
\hline & $\mathrm{H}$ & \multicolumn{3}{|c|}{5,696} & \multicolumn{5}{|c|}{2,483} \\
\hline & $p$ & \multicolumn{3}{|c|}{0,058} & \multicolumn{5}{|c|}{0,648} \\
\hline
\end{tabular}

Сравнительный анализ, проведённый с помощью критерия Краскела-Уоллиса, показал, что выявленные различия статистически не значимы, так как уровень значимости во всех случаях превышает 0,05 (выделено в таблице 3). Следовательно, нет оснований для отклонения нулевой гипотезы о равенстве средних. Таким образом, между студентами, изучающими разные иностранные языки (английский - немецкий - французский), а также между студентами, по разному оценивающими собственный уровень владения иностранным языком (А1 - A2 - B1 - В2 - С1C2), нет статистически значимых различий по уровню перфекционизма и его составляющих.

\section{4. Сила эффекта}

Ещё одной полезной мерой оценки наблюдаемых различий служит сила эффекта - мера, активно применяемая в современных зарубежных исследованиях, в том числе лингводидактических. Известно, что сила эффекта определённым образом конкурирует со статистической значимостью как инструмент оценки эмпирических результатов [12, с. 136]. Эта мера позволяет не упустить важное отличие, даже если оно не обладает статистической значимостью (поскольку во многом зависит от размера выборки и изменчивости данных). Поэтому результаты, полученные в данном исследовании, были оценены и по силе эффекта (см. таблицу 4). В сфере образования в качестве показателя силы эффекта чаще всего используется эффект Коэна (Cohen’s D). При интерпретации полученных результатов мы опирались на рекомендации Дж. Хэтти для сферы образования: слабый эффект $(\mathrm{D}<0,2)$, средний $(0,2<\mathrm{D}<0,4)$, сильный $(0,4<\mathrm{D}<0,6)[11$, c. 9$]$.

Таблица 4.

Сравнение выявленных отличий в степени перфекционизма по силе эффекта

\begin{tabular}{|c|c|c|c|}
\hline Виды перфекционизма & Сравниваемые группы & Cohen's D & Интерпретация \\
\hline \multirow{5}{*}{} & en - de & 0,164 & слабый эффект \\
\cline { 2 - 4 } & en - fr & 0,148 & слабый эффект \\
\cline { 2 - 4 } & $\mathrm{de}-\mathrm{fr}$ & 0,336 & средний эффект \\
\cline { 2 - 4 } & $\mathrm{A} 1-\mathrm{A} 2$ & $\mathbf{0 , 4 8 7}$ & сильный эффект \\
\cline { 2 - 4 } & $\mathrm{A} 1-\mathrm{B} 1$ & 0,068 & слабый эффект \\
\cline { 2 - 4 } & $\mathrm{A} 1-\mathrm{B} 2$ & 0,088 & слабый эффект \\
\cline { 2 - 4 } & $\mathrm{A} 1-\mathrm{C} 1-\mathrm{C} 2$ & 0,008 & очень слабый эффект \\
\cline { 2 - 4 } & $\mathrm{A} 2-\mathrm{B} 1$ & $\mathbf{0 , 4 7 4}$ & сильный эффект \\
\cline { 2 - 4 } & $\mathrm{A} 2-\mathrm{B} 2$ & 0,219 & средний эффект \\
\cline { 2 - 4 } & $\mathrm{A} 2-\mathrm{C} 1-\mathrm{C} 2$ & 0,337 & средний эффект \\
\cline { 2 - 4 } & $\mathrm{B} 1-\mathrm{B} 2$ & 0,145 & слабый эффект \\
\cline { 2 - 4 } & $\mathrm{B} 1-\mathrm{C} 1-\mathrm{C} 2$ & 0,066 & слабый эффект \\
\cline { 2 - 4 } & $\mathrm{B} 2-\mathrm{C} 1-\mathrm{C} 2$ & 0,081 & слабый эффект \\
\hline \multirow{4}{*}{$\begin{array}{l}\text { Перфекционизм, } \\
\text { ориентированный на себя }\end{array}$} & $\mathrm{en}-\mathrm{de}$ & 0,022 & слабый эффект \\
\cline { 2 - 4 } & $\mathrm{en}-\mathrm{fr}$ & 0,031 & слабый эффект \\
\hline
\end{tabular}


Продолжение таблицы 4.

\begin{tabular}{|c|c|c|c|}
\hline Виды перфекционизма & Сравниваемые группы & Cohen's D & Интерпретация \\
\hline \multirow{11}{*}{$\begin{array}{l}\text { Перфекционизм, } \\
\text { ориентированный на себя }\end{array}$} & $\mathrm{de}-\mathrm{fr}$ & 0,054 & слабый эффект \\
\hline & $\mathrm{A} 1-\mathrm{A} 2$ & 0,549 & сильный эффект \\
\hline & $\mathrm{A} 1-\mathrm{B} 1$ & 0,232 & средний эффект \\
\hline & $A 1-B 2$ & 0,143 & слабый эффект \\
\hline & $\mathrm{A} 1-\mathrm{C} 1-\mathrm{C} 2$ & 0,023 & слабый эффект \\
\hline & $\mathrm{A} 2-\mathrm{B} 1$ & 0,657 & очень сильный эффект \\
\hline & $\mathrm{A} 2-\mathrm{B} 2$ & 0,201 & средний эффект \\
\hline & $A 2-C 1-C 2$ & 0,385 & средний эффект \\
\hline & $\mathrm{B} 1-\mathrm{B} 2$ & 0,355 & средний эффект \\
\hline & $\mathrm{B} 1-\mathrm{C} 1-\mathrm{C} 2$ & 0,186 & слабый эффект \\
\hline & $\mathrm{B} 2-\mathrm{C} 1-\mathrm{C} 2$ & 0,161 & слабый эффект \\
\hline \multirow{13}{*}{$\begin{array}{l}\text { Перфекционизм, } \\
\text { ориентированный на других }\end{array}$} & en - de & 0,018 & слабый эффект \\
\hline & en $-\mathrm{fr}$ & 0,175 & слабый эффект \\
\hline & $\mathrm{de}-\mathrm{fr}$ & 0,213 & средний эффект \\
\hline & $\mathrm{A} 1-\mathrm{A} 2$ & 0,247 & средний эффект \\
\hline & $\mathrm{A} 1-\mathrm{B} 1$ & 0,324 & средний эффект \\
\hline & $\mathrm{A} 1-\mathrm{B} 2$ & 0,461 & сильный эффект \\
\hline & $\mathrm{A} 1-\mathrm{C} 1-\mathrm{C} 2$ & 0,556 & сильный эффект \\
\hline & $A 2-B 1$ & 0,085 & слабый эффект \\
\hline & $\mathrm{A} 2-\mathrm{B} 2$ & 0,260 & средний эффект \\
\hline & $\mathrm{A} 2-\mathrm{C} 1-\mathrm{C} 2$ & 0,343 & средний эффект \\
\hline & $\mathrm{B} 1-\mathrm{B} 2$ & 0,189 & слабый эффект \\
\hline & $\mathrm{B} 1-\mathrm{C} 1-\mathrm{C} 2$ & 0,264 & средний эффект \\
\hline & $\mathrm{B} 2-\mathrm{C} 1-\mathrm{C} 2$ & 0,056 & слабый эффект \\
\hline \multirow{13}{*}{$\begin{array}{l}\text { Социально предписанный } \\
\text { перфекционизм }\end{array}$} & en-de & 0,357 & средний эффект \\
\hline & $e n-f r$ & 0,132 & слабый эффект \\
\hline & $\mathrm{de}-\mathrm{fr}$ & 0,531 & сильный эффект \\
\hline & $\mathrm{A} 1-\mathrm{A} 2$ & 0,728 & очень сильный эффект \\
\hline & $\mathrm{A} 1-\mathrm{B} 1$ & 0,466 & сильный эффект \\
\hline & $\mathrm{A} 1-\mathrm{B} 2$ & 0,485 & сильный эффект \\
\hline & $\mathrm{A} 1-\mathrm{C} 1-\mathrm{C} 2$ & 0,640 & очень сильный эффект \\
\hline & $\mathrm{A} 2-\mathrm{B} 1$ & 0,157 & слабый эффект \\
\hline & $\mathrm{A} 2-\mathrm{B} 2$ & 0,030 & слабый эффект \\
\hline & $\mathrm{A} 2-\mathrm{C} 1-\mathrm{C} 2$ & 0,037 & слабый эффект \\
\hline & $\mathrm{B} 1-\mathrm{B} 2$ & 0,096 & слабый эффект \\
\hline & $\mathrm{B} 1-\mathrm{C} 1-\mathrm{C} 2$ & 0,179 & слабый эффект \\
\hline & $\mathrm{B} 2-\mathrm{C} 1-\mathrm{C} 2$ & 0,059 & слабый эффект \\
\hline
\end{tabular}

По группам, изучающим разные языки, сильный эффект (значимые различия) наблюдается между студентами, изучающими немецкий и французский $(\mathrm{D}=0,531)$, то есть студенты, изучающие немецкий, ощущают требования извне более остро, чем студенты, изучающие французский. Возможно, это связано с тем, что требования преподавателей немецкого языка действительно строже, чем преподавателей других языковых отделений.

По самооценке уровня владения изучаемым языком наиболее сильные различия проявились: по общему перфекционизму - между уровнями $\mathrm{A} 1$ и $\mathrm{A} 2(\mathrm{D}=0,487)$, а также $\mathrm{A} 2$ и $\mathrm{B} 1(\mathrm{D}=0,474)$. То есть студенты с уровнем А2 наиболее сильно отличаются от студентов на соседних с ними уровнях.

Что касается составляющих перфекционизма, то следует отметить отличие в части перфекционизма, ориентированного на себя, - очень сильное между уровнями $\mathrm{A} 2$ и $\mathrm{B} 1(\mathrm{D}=0,657)$ и сильное между уровнями $\mathrm{A} 1$ и $\mathrm{A} 2(\mathrm{D}=0,549)$. По уровню перфекционизма, ориентированного на других, сильно отличаются уровни $\mathrm{A} 1$ и $\mathrm{B} 2(\mathrm{D}=0,461)$, а также $\mathrm{A} 1$ и $\mathrm{C} 1 / \mathrm{C} 2(\mathrm{D}=0,556)$. По уровню социально предписанного перфекционизма уровень A1 отличается от других очень сильно или сильно $(0,466 \leq \mathrm{D} \geq 0,728)$, но все остальные уровни слабо отличаются друг от друга.

Таким образом, оценка силы эффекта позволила заметить различия, которые требуют более пристального внимания при продолжении исследования. В частности, проявилась тенденция от- 
личия элементарных уровней владения языком (А1 и А2) от остальных. Возможно, это связано с тем, что пройдя первый этап изучения языка, студенты начинают пучше понимать собственные ошибки и пробелы в знаниях, а также лучше осознают свою зону ближайшего языкового развития.

\section{Заключение}

Полученные результаты в целом согласуются с результатами других исследований $[5 ; 19 ; 10$; 4]. Однако наше исследование проводилось со студентами одного университета и даже одного института. Выявленная специфика социально предписанного перфекционизма, по-видимому, характерна для конкретной выборки и вряд ли может быть экстраполирована на студентов других университетов. Чтобы обобщать результаты, в будущем возможно изучить студентов разных институтов или даже разных вузов. Кроме того, в качестве одной из переменных использовалась самооценка уровня владения иностранным языком. Проследить более точные закономерности позволит использование объективной оценки уровня владения языком, например, одного из международных стандартизированных тестов. Подобный подход уже был реализован в ВШЭ [15, c. 139], где использовался балл IELTS (что применимо только к английскому языку). В перспективе будет полезно сравнить данные по разным изучаемым языкам.

Эмпирические данные исследования позволили проследить определённые тенденции в степени перфекционизма и отдельных его видов. Интересно, что наблюдаются определённые различия по уровню владения языком. Однако настоящее исследование было перекрёстным, то есть выводы о причинно-следственных связях невозможны. Но его результаты позволяют сформулировать более точные гипотезы о влиянии перфекционизма на успешность изучения иностранных языков и продолжить работу в этом направлении в формате лонгитюдного исследования.

Статья публикуется впервые.

(C) Савина О.Ю., 2020

\section{Список литературы}

1. Грачева И.И. Адаптация методики «Многомерная шкала перфекционизма» П. Хьюитта и Г. Флетта // Психологический журнал. 2006. № 6. С. 73-81.

2. Грачева И.И. Многомерная шкала перфекционизма Хьюитта-Флетта [Электронный ресурс]. - URL: https://sites.google. com/site/test300m/msp (дата доступа 29.06.2019).

3. Золотарева А.А. Краткий дифференциальный тест перфекционизма: проверка кросс-культурной устойчивости факторной структуры и психометрических характеристик // Культурно-историческая психология. 2018. № 1. С. 107-115.

4. Карловская Н.Н. Взаимосвязь переживания счастья и перфекционизма у студентов с разным уровнем академической успеваемости / Н.Н. Карловская, А.С. Сысоева // Вестник Омского университета. Серия «Психология». 2009 . № 2. С. $4-14$.

5. Киселева Л.Б. Уровень перфекционизма у студентов с различной успеваемостью по английскому языку // Современные исследования социальных проблем. 2015. № 11 (55). С. 427-441.

6. Многомерная шкала перфекционизма Хьюитта-Флетта [Электронный ресурс]. - URL: https://psylab.info/Многомерная_ шкала_перфекционизма_Хьюитта-Флетта (дата доступа 23.06.2019).

7. Наследов А.Д. Адаптация «Опросника перфекционизма» для диагностики перфекционистских установок студентов первого курса технических вузов / А.Д. Наследов, Л.Б. Киселева // Вестник Санкт-Петербургского университета. Серия 16. Психология. Педагогика. 2016. № 3. С. 44-64.

8. Пермякова Т.М. Систематизация отечественных исследований и кросс-культурные перспективы изучения перфекционизма / Т.М. Пермякова, М.С. Шевелева // Вестник Пермского университета. Философия. Психология. Социология. 2015. № 2 (22). С. 65-73.

9. Ясная В.А. Современные модели перфекционизма / В.А. Ясная, С.Н. Ениколопов // Психологические исследования. 2013. № 6 (29). [Электронный ресурс]. - URL: http://psystudy.ru/index.php/num/2013v6n29/826-yasnaya29.html (дата доступа 23.06.2020).

10. Farag I. Perfectionism and English Learners' Self-efficacy // ECPS - Educational Cultural and Psychological Studies. 2020. No. 21. P. 87-109.

11. Hattie J. Visible learning for teachers / J. Hattie. London, New York: Routledge, 2012. 269 p. 
12. Larson-Hall J. Reporting and interpreting quantitative research findings: What gets reported and recommendations for the field / J. Larson-Hall, L. Plonsky // Language Learning. 2015. No. 65. P. 127-159.

13. Mahasneh A.M. The level of Multidimensional Perfectionism and Motivational Orientation among Undergraduate Students / A.M. Mahasneh, A.F. Alwan, T.M. Al-Rawwad // Psychology in Russia: State of the Art. 2019. No. 2. P. 94-114.

14. Maintaining the self? Exploring the connections between students' perfectionistic profiles, self-worth contingency, and achievement goal orientations / Je. Ståhlberg, H. Tuominen, A.-T. Pulkka, et al. // Personality and Individual Differences. 2019. No. 151. P. 1-11.

15. Perfectionism as a Predictor of Anxiety in Foreign Language Classrooms among Russian College Students / K. Wang, T. Permyakova, M. Sheveleva, et al. // Journal of Educational, Cultural and Psychological Studies. 2018. No. 18. P. 127-146.

16. Positive and negative perfectionism / L.A. Terry-Short, R.G. Owens, P.D. Slade, et al. // Personality and Individual Differences. 1995. No. 5. P. 663668.

17. Rice K.G. The short form of the revised almost perfect scale / K.G. Rice, C.M.E. Richardson, S. Tueller // Journal of personality assessment. 2014. No. 3. P. 368-379.

18. The Multidimensional Perfectionism Scale: Reliability, Validity, and Psychometric Properties in Psychiatric Samples / P.L. Hewitt, G.L. Flett, W. Turnbull-Donovan, et al. // Psychological Assessment: A Journal of Consulting and Clinical Psychology. 1991. Vol. 3, No. 3. P. 464-468.

19. The Relationship between Perfectionism and Academic Achievement, Depression and Anxiety / H. Roohafza, H. Afshar, M. Sadeghi, et al. // Iranian Journal of Psychiatry and Behavioral Sciences. 2010. Vol. 4, No. 2. P. 31-36.

20. Wang K.T. Assessing perfectionism in Russia: Classifying perfectionists with the Short Almost Perfect Scale / K.T. Wang, T.M. Permyakova, M.S. Sheveleva // Personality and Individual Differences. 2016. No. 92. P. 174-179.

\section{References}

1. Gracheva, I.I. Adaptatsiia metodiki «Mnogomernaia shkala perfektsionizma» P. Kh'iuitta i G. Fletta [Adaptation of "Multidimensional Perfectionism Scale" Technique by P. Hewitt and G. Flett]. Psikhologicheskii zhurnal [Psychological Journal], no. 6, 2006, pp. 73-81.

2. Gracheva, I.I. Mnogomernaia shkala perfektsionizma Kh'iuitta-Fletta [The Multidimensional Perfectionism Scale of Hewitt and Flett], sites.google.com/site/test $300 \mathrm{~m} / \mathrm{msp}$ (Accessed 29 June 2019).

3. Zolotareva, A.A. Kratkii differentsial'nyi test perfektsionizma: proverka kross-kul'turnoi ustoichivosti faktornoi struktury i psikhometricheskikh kharakteristik [Brief Differential Perfectionism Inventory: Checking Cross-Cultural Stability of the Factor Structure and Psychometric Characteristics]. Kul'turno-istoricheskaia psikhologiia [Cultural-Historical Psychology], no. 1, 2018, pp. 107-115.

4. Karlovskaia, N.N., Sysoeva, A.S. Vzaimosviaz' perezhivaniia schast'ia i perfektsionizma u studentov s raznym urovnem akademicheskoi uspevaemosti [The Relationship between Happiness and Perfectionism among Students with Different Levels of Academic Achievement]. Vestnik Omskogo universiteta. Seriia "Psikhologiia» [Herald of Omsk University. Series "Psychology"], no. 2, 2009, pp. 4-14.

5. Kiseleva, L.B. Uroven' perfektsionizma u studentov s razlichnoi uspevaemost'iu po angliiskomu iazyku [Perfectionism Level of Students with Various Progress in the English Language]. Sovremennye issledovaniia sotsial'nykh problem [Modern Studies of Social Issues], no. 11 (55), 2015, pp. 427-441.

6. Mnogomernaia shkala perfektsionizma Kh'iuitta-Fletta [The Multidimensional Perfectionism Scale of Hewitt and Flett], psylab. info/Mnogomernaia_shkala_perfektsionizma_Kh'iuitta-Fletta (Accessed 23 June 2019).

7. Nasledov, A.D., Kiseleva, L.B. Adaptatsiia «Oprosnika perfektsionizma» dlia diagnostiki perfektsionistskikh ustanovok studentov pervogo kursa tekhnicheskikh vuzov [Adaptation of the "Perfectionism Questionnaire" to Diagnose Perfectionist Attitudes among First-Year Technical University Students]. Vestnik Sankt-Peterburgskogo universiteta. Seriia 16. Psikhologiia. Pedagogika [Vestnik of Saint Petersburg University. Psychology], no. 3, 2016, pp. 44-64.

8. Permiakova, T.M., Sheveleva, M.S. Sistematizatsiia otechestvennykh issledovanii i kross-kul'turnye perspektivy izucheniia perfektsionizma [Perfectionism Studies in Russia and their Cross-cultural Prospects]. Vestnik Permskogo universiteta. Filosofiia. Psikhologiia. Sotsiologiia [Perm University Herald. Series Philosophy. Psychology. Sociology], no. 2 (22), 2015, pp. 65-73.

9. Iasnaia, V.A., Enikolopov, S.N. Sovremennye modeli perfektsionizma [The Current Models of Perfectionism]. Psikhologicheskie issledovaniia [Psychological Studies], no. 6 (29), 2013, psystudy.ru/index.php/num/2013v6n29/826-yasnaya29.html (Accessed 23 June 2020).

10. Farag, Islam. "Perfectionism and English Learners' Self-efficacy". ECPS - Educational Cultural and Psychological Studies, no. 21, 2020, pp. 87-109.

11. Hattie, John. Visible learning for teachers. London, New York: Routledge, 2012.

12. Larson-Hall, Jenifer, and Luke Plonsky. "Reporting and Interpreting Quantitative Research Findings: What gets reported and Recommendations for the Field”. Language Learning, no. 65, 2015, pp. 127-159.

13. Mahasneh, Ahmad M., et al. ,The level of Multidimensional Perfectionism and Motivational Orientation among Undergraduate Students". Psychology in Russia: State of the Art, no. 2, 2019, pp. 94-114.

14. Ståhlberg, Jenny, et al. "Maintaining the self? Exploring the Connections between Students' Perfectionistic Profiles, Self-worth Contingency, and Achievement Goal Orientations". Personality and Individual Differences, no. 151, 2019, pp. 1-11.

15. Wang, Kenneth, et al. "Perfectionism as a Predictor of Anxiety in Foreign Language Classrooms among Russian College Students". Journal of Educational, Cultural and Psychological Studies, no. 18, 2018, pp. 127-146.

16. Terry-Short, L.A., et al. "Positive and Negative Perfectionism. Personality and Individual Differences, no. 5, 1995, pp. 663668. 
17. Rice, Kenneth G., et al. „The Short Form of the Revised Almost Perfect Scale”. Journal of personality assessment, no. 3, 2014, pp. 368379.

18. Hewitt, Paul L., et al. „The Multidimensional Perfectionism Scale: Reliability, Validity, and Psychometric Properties in Psychiatric Samples". Psychological Assessment: A Journal of Consulting and Clinical Psychology, vol. 3, no. 3, 1991, pp. 464-468.

19. Roohafza, Hamidreza, et al. "The Relationship between Perfectionism and Academic Achievement, Depression and Anxiety". Iranian Journal of Psychiatry and Behavioral Sciences, vol. 4, no. 2, 2010, pp. 31-36.

20. Wang, Kenneth T., et al. „Assessing Perfectionism in Russia: Classifying Perfectionists with the Short Almost Perfect Scale”. Personality and Individual Differences, no. 92, 2016, pp. 174-179.

\section{Сведения об авторе:}

Савина Ольга Юрьевна - доцент кафедры немецкой филологии ТюмГУ, кандидат филологических наук (Россия, Тюмень). Сфера научных и профессиональных интересов: компьютерная лингводидактика, корпусная лингвистика.

E-mail: osawina@gmail.com

Конфликт интересов: Автор заявляет об отсутствии конфликта интересов.

\section{About the authors:}

Olga Yu. Savina - Associate Professor at the Department of German Philology, University of Tyumen, Candidate of Philological Sciences (Tyumen, Russia). Spheres of research and professional interest: Computerassisted language learning, Corpus linguistics.

E-mail: osawina@gmail.com

Conflicts of interest: The author declares absences of conflicts of interest. 